dine sensitivity of gating current in the presence and absence of $\beta$-subunits.

Because $\beta$-subunit binding to the $\alpha_{1-}$ subunit modulates the gating of the $\mathrm{Ca}^{2+}$ channel, it will be interesting to see whether the interaction between the subunits can be altered by phosphorylation or other influences such as G proteins. Cardiac $\mathrm{Ca}^{2+}$ current is increased by $\beta$-adrenergic stimulation ${ }^{10}$, and the $\beta$-subunit may be needed for this modulation $^{11}$. If so, the present line of research may lead to a molecular understanding of the original example of a voltage-dependent ion channel whose behaviour is modulated by neurotransmitters.

Bruce Bean is in the Department of Neurobiology, Harvard Medical School, 220 Longwood Avenue, Boston, Massachusetts 02115, USA.

\title{
Coming to an unsticky end
}

\section{RobertF. Brady Jr}

THE non-stick surface - a surface that resists or even repels clinging matter and never needs cleaning - may be the chemical equivalent of the perpetual motion machine: earnestly desired, but unattainable. Creating such a surface requires careful control of the composition,

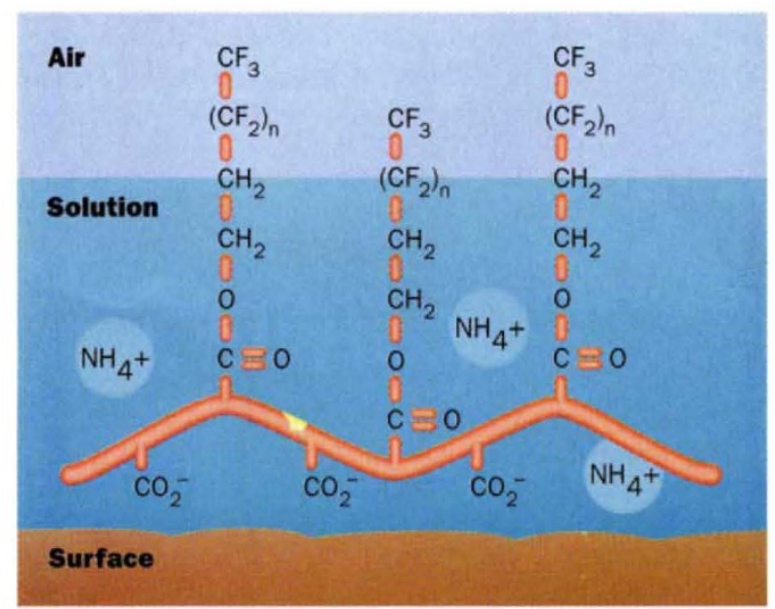

Coming unstuck - the favoured conformation of a fluorinated surfactant directs hydrophobic groups towards the air-water interface and ionic groups towards the substrate.

free energy, texture and electrical properties of the surface, and these parameters must be optimized for each application. Current research on non-stick coatings focuses on minimizing their surface free energy, for this is believed to be the dominant factor controlling anti-adhesive behaviour. On page 39 of this issue, Schmidt and co-workers ${ }^{1}$ report an ingenious water-based polymerization technique to produce a hydrophobic coating with an unusually low surface energy, about $11-16 \mathrm{~mJ} \mathrm{~m}^{-2}$. The hard, clear coating is not wetted by solvents and does not attach to adhesives.

Non-stick coatings are crucial for many applications, including peel-off backings for self-stick labels ${ }^{2}$, prosthetic devices that do not accumulate biological debris, bearings and valves, non-toxic foulingrelease coatings for ships' hulls ${ }^{3}$, stainresistant fabrics and self-lubricating sur- faces. The coatings must, of course, be safe to manufacture and use; in our drive for cleanliness we do not wish to taint either our bodies or our environment.

In a new approach to coatings with low-energy surfaces, Schmidt and coworkers polymerize perfluorooctyl acrylate or perfluorooctyl methacrylate esters with monomers containing ionizable functional groups to create a fluorinated polymeric surfactant. In solution this polymer spontaneously organizes itself to present the fluorinated groups to the air-water interface and the ionic groups to the substrate (see figure). At this temporary stage the coating is not wetted by liquid hydrocarbons, but redissolves in polar liquids because ionic groups remain unreacted.

The authors prepare robust coatings by reacting this polymer with a watercompatible crosslinking polymer; they use a polymer with pendant oxazoline rings for this purpose. A mixture of the two polymers is stable in a water-glycol solution, but forms a sturdy crosslinked film when applied to a surface and cured at $130{ }^{\circ} \mathrm{C}$ for an hour. Synthetic adhesives do not stick to the film, and it is not wetted or attacked by common solvents. A second coat of the same material adheres well, however, because fluorinated groups collect at the interface where they align with the fluorinated groups in the first coating.

The concept that a low-energy surface is best achieved by closely packing and orienting perfluoroalkyl $\left(\mathrm{R}_{\mathrm{F}}\right)$ groups is evolutionary, rather than revolutionary. Surface energy is known to be related to the functional groups at the polymer surface ${ }^{4}$; among polymers in common use, the hydrocarbon-based poly(ethylene) and poly(propylene) have the lowest surface energies. But when fluorine is substituted for hydrogen, the surface energy is reduced further. Electrons are held closely and tightly about the fluorine nucleus and cannot be shared or easily polarized, whereas the electron density around the hydrogen nucleus is mostly in the covalent bond, leaving the nucleus unshielded and able to induce weak bonds. Thus substitution of fluorine causes the surface energy to decrease in the order $-\mathrm{CH}_{2-}>-\mathrm{CH}_{3}>-\mathrm{CF}_{2-}>$ $-\mathrm{CF}_{3}$. This is readily observed in the surface energies of polymers with surfaces composed of ordered single groups such as poly(ethylene) $\left(33.7 \mathrm{~mJ} \mathrm{~m}^{-2}\right)$, poly(din.ethylsiloxane) (21.2), poly(tetrafluoroethylene) (PTFE) (18.6) and poly[di(3,3,3-trifluoropropyl)siloxane] (6.0).

Many once believed that PTFE, the most familiar of the non-stick polymers, would be the answer to their prayers, but its usefulness is limited. PTFE is not very easily processed because it is not dissolved, softened or even wetted by common solvents. It is effective in cookware and some other applications but it accumulates all types of marine fouling with astonishing speed because of its porosity - marine adhesives invade cavities in the surface and cure inside them, creating a secure mechanical interlock ${ }^{5}$. There is a need for a coatings technology that can deposit a non-porous PTFE-like surface wherever desired. This technology will have to comply with a host of contemporary environmental and human health regulations such as the reduction or elimination of air-polluting organic solvents in favour of water-based technologies. Schmidt and co-workers have made an impressive move in this direction.

Producing polymers with a large number of $R_{F}$ groups is relatively easy, but causing these polymers to form ordered films with the $R_{F}$ groups aligned and closely packed on the surface is far more difficult. The authors use ethylene glycol (or another polar liquid) to plasticize the film, keeping it pliant as crosslinking progresses and providing time for systematic orientation of the $R_{F}$ groups. Polar liquids also improve surface smoothness, another important contributor to non-stick behaviour. Surfaces in air, water and hexadecane, studied using scanning force microscopy, show a peak-to-valley roughness of $0.4-1.3 \mathrm{~nm}$ which is independent of environment or crosslink density.

After the $R_{F}$ groups form on the surface, they may not stay there, for liquids on the film may cause the $R_{F}$ groups to migrate into the film. Schmidt and coworkers use contact angle studies with hexadecane and water to evaluate the orientation and mobility of the $\mathrm{R}_{\mathrm{F}}$ groups and to obtain an estimate of the surface free energy. A series of coatings is studied 


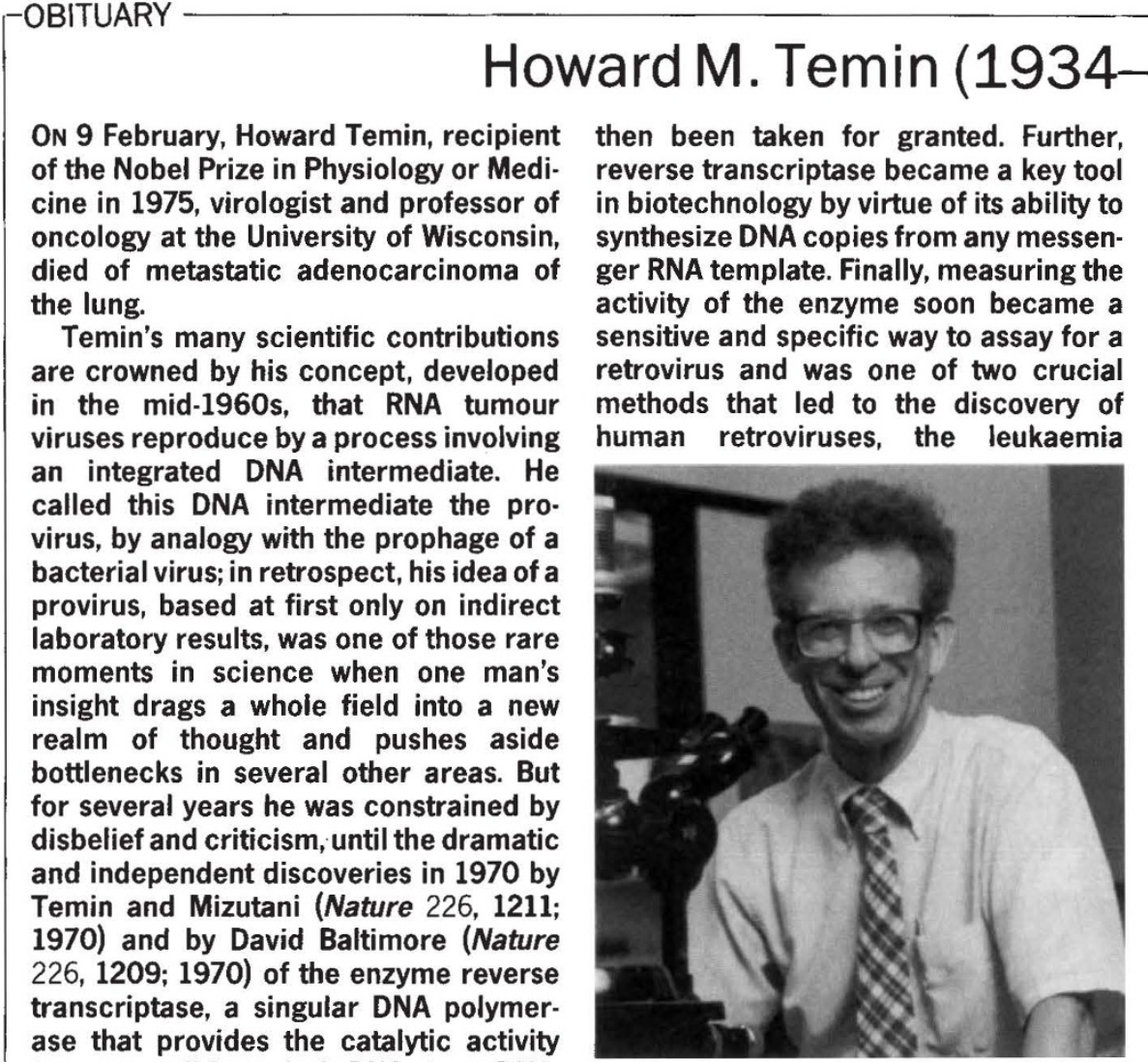

for transcribing viral RNA into DNA. The enzyme of Temin and Mizutani was from a chicken retrovirus whereas Baltimore's was from a mouse leukaemia retrovirus, but it was soon learned that reverse transcriptase is carried by every infectious RNA tumour virus.

The way was now open to determine the replication cycle of these cancercausing animal viruses and the strategies they use to transform cells, but this was only the start of the impact of reverse transcriptase on molecular biology. The unidirectional flow of genetic infor. mation, DNA to RNA to protein, had until then been taken for granted. Further, reverse transcriptase became a key tool in biotechnology by virtue of its ability to DNA copies from any messensensitive and specific way to assay for a retrovirus and was one of two crucial leukaemia

Howard Temin, Nobel prize winner in 1975.

viruses (HTLV-I and HTLV-II) and the human immunodeficiency virus responsible for AIDS.

Temin began his postdoctoral fellowship at Cal Tech with Harry Rubin and Renato Dulbecco in 1956 (Dulbecco and Baltimore shared the 1975 Nobel prize with Temin). He started studying the transformation of cells by the Rous (avian) sarcoma retrovirus when he realized that this in vitro phenomenon, then just described by scientists at Rutgers University, was an opening to underactivity of the enzyme soon became a standing cellular alteration, an interest he maintained throughout his career. In 1960 he moved to Madison to join the cancer research centre at the McArdle Laboratory in the University of Wisconsin, where he remained a member of this renowned group. In recent years he turned his attention to mechanisms of generating variation in retroviruses, central to the problem of drug resistance and vaccine development for AIDS. He continued to be active as a teacher, researcher and advisor to the National Cancer Institute (as a member of the National Cancer Advisory Board) until only weeks before his untimely death.

Howard Temin's mind held a rare combination of creativity and penetrating, analytical insights, and his judgement was a rare combination of compassion and independent objectivity. In an era when biological laboratories have grown in size, needs and complexity, his team maintained a focus on individual creativity. Despite the modern pressures on families, he was a devoted family man - to his wife, Rayla Greenberg Temin (geneticist at the University of Wisconsin), and to his daughters, Sarah and Miriam. Finally, at a time when biomedical scientists in the United States are more and more involved in entrepreneurial adventures, Temin remained where he began, the pure academician. Once when I asked him why, his reply was a typical Teminism: "With all those experiments going on out there, there must be at least one control!".

Robert C. Gallo

Robert C. Gallo is at the National Cancer Institute, National Institutes of Health, Bethesda, Maryland 20892, USA. in which the ratio of fluorinated polymeric surfactant to crosslinking agent is varied between $0.3: 1$ and $3: 1$. As the films become more highly crosslinked, the concentration of $R_{F}$ groups at the surface decreases, and these groups are less able to reorient themselves. The surface free energy lies between 11 and $16 \mathrm{~mJ} \mathrm{~m}^{-2}$, indicating that the surface is indeed composed of a preponderance of $R_{F}$ groups. $\mathrm{X}$-ray data are consistent with layers of $\mathrm{R}_{\mathrm{F}}$ groups about $8 \mathrm{~nm}$ thick.

The new synthetic scheme is versatile enough to allow production of surfaces with a range of energies. A series of coatings with tailored surface energies could be made by varying the monomer ratios in the reactants, perhaps using non-fluorinated monomers when higher surface energy is desired, and used to isolate and explore the influence of surface free energy, which is widely believed to be the primary determinant of ad- hesion. Although synthetic adhesives show a reasonable (but not perfect) inverse relationship between bonding strength and surface energy, natural glycoproteins and polysaccharides show the least adhesion within a range of surface energy which is near, but does not include, the lowest values. Baier and Meyer $^{6}$ recommend 20 to $30 \mathrm{~mJ} \mathrm{~m}^{-2}$ as the optimum range for the free energy of a surface designed to shed biofouling and say that efforts to reduce the surface energy below this range are counterproductive.

Before we conclude that the ultimate non-stick coating is at hand, silicone and urethane chemists must have their innings. Compared with a carbon backbone, the $\mathrm{O}-\mathrm{Si}-\mathrm{O}$ backbone has a more open structure, with a flatter angle and a longer bond length; available surface-active groups on a silicone backbone adopt the lowest surface-energy configuration much more readily. Silicone coatings will need improved mechanical strength for some applications. Elastomeric urethane coatings, however, combine backbone flexibility with good mechanical properties. My view is that the consummate non-stick coating will have a silicone backbone, but I'm not yet prepared to bet whether it will contain fluorine.

Robert F. Brady Jris in the Coatings Section, US Naval Research Laboratory, Washington DC 20375, USA.

1. Schmidt, D. L. et al. Nature 368, 39-41 (1994)

2. Owen, M. J.J. Coatings Technol. 53 (679), 49-53 (1981)

3. Brady, R. F. Jr, Griffith, J. R., Love, K. S. \& Field, D. E. J. Coatings Technol. 59 (755), 113-119 (1987)

4. Zisman, W. A. in Contact Angle, Wettability, and Adhesion Adv. Chem. Ser. 43, 1-51 (Am. Chem. Soc., Washington DC, 1964).

5. Saroyan, J. R., Lindner, E., Dooley, C. A. \& Bleile, H. R. Indust. Engng Chem., Prod. Res. Devel. 9, 123-128 (1970)

6. Baier, R. E. \& Meyer, A. E. Biofouling 6, 165-180 (1992). 